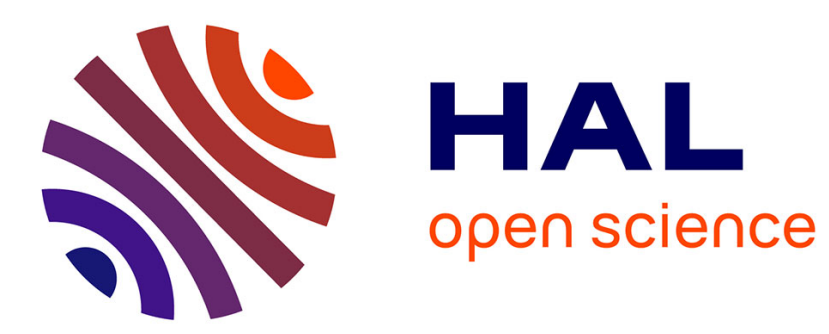

\title{
Identification of nonmonotonic behaviors and stick-slip transition in liquid crystal polymers
}

\author{
Caroline Pujolle-Robic, Laurence Noirez
}

\section{To cite this version:}

Caroline Pujolle-Robic, Laurence Noirez. Identification of nonmonotonic behaviors and stick-slip transition in liquid crystal polymers. Physical Review E , 2003, 10.1103/PhysRevE.68.061706 . hal01361937

\section{HAL Id: hal-01361937 \\ https://hal.science/hal-01361937}

Submitted on 7 Sep 2016

HAL is a multi-disciplinary open access archive for the deposit and dissemination of scientific research documents, whether they are published or not. The documents may come from teaching and research institutions in France or abroad, or from public or private research centers.
L'archive ouverte pluridisciplinaire HAL, est destinée au dépôt et à la diffusion de documents scientifiques de niveau recherche, publiés ou non, émanant des établissements d'enseignement et de recherche français ou étrangers, des laboratoires publics ou privés. 


\title{
Identification of nonmonotonic behaviors and stick-slip transition in liquid crystal polymers
}

\author{
Caroline Pujolle-Robic and Laurence Noirez* \\ Laboratoire Léon Brillouin (CEA-CNRS), CE Saclay, 91191 Gif sur Yvette, France
}

(Received 26 February 2003; published 31 December 2003)

\begin{abstract}
The recent identification of shear-induced phases in the isotropic melts of liquid crystal polymers shows that these materials are expected to display original nonlinear behaviors. We have investigated the flow behavior of a nematic sidechain polymer above its isotropic-nematic transition temperature. Nonlinear rheology and birefringence measurements indicate the appearance, above a critical shear rate, of the shear-induced isotropicnematic phase transition. The rheological behavior of this induced phase is characterized by undamped timeperiodic shear stress oscillations. These sustained oscillations are interpreted in terms of a stick-slip mechanism alternating high-friction static state and low-friction kinetic state.

DOI: 10.1103/PhysRevE.68.061706

PACS number(s): 83.80.Xz, 47.20.Hw, 83.50.Ax, 64.70.Md
\end{abstract}

Polymers are non-Newtonian fluids [1] whereas liquid crystals do not behave as simple fluids close to phase transitions [2]. When these two complex fluids are coupled to form a melt of sidechain liquid crystal polymers (SCLCPs), the resulting rheological behavior is expected to be peculiar. The very first flow studies [3] have indeed indicated that SCLCP melts display strong nonlinear behaviors above the isotropicnematic transition temperature $\left(T_{\mathrm{NI}}\right)$. This behavior looks similar to the well-studied shear-induced behavior of giant micelle solutions which display a shear-induced $I-N$ transition above $T_{\mathrm{NI}}[4,5]$. The SCLCP shear-induced transition was revealed by flow birefringence and via the existence of a stress plateau in the stress versus shear rate curve. The stress plateau can be explained by entering an unstable flow region; above a critical shear rate, the region is characterized by a decreasing stress with increasing shear rate. The system is then supposed to phase separate into homogeneous bands (shear banding) to maintain the imposed shear rate [5]. The existence of such nonequilibrium states opens the question of identification of the coupling parameters associated with the critical shear rate. Clearly, the shear induced SCLCP critical times are not associated with the lifetime of the pretransitional fluctuations, suggesting a coupling with slower time scales which could be rather consistent with the existence of macroscopic heterogeneities as proposed by Collin et al. [6]. The shear-induced phase conformation of the polymer main chain was also determined using small angle neutron scattering. For a LC polymer characterized in the equilibrium nematic phase by a perpendicular main chain/mesogen coupling (oblate conformation), we observed that the initially perpendicular coupling is inverted in the shear-induced nematic phase to a parallel coupling with the main-chain conformation becoming prolate [3]. This structural rearrangement can be proposed as a working hypothesis to explain the appearance of shear-induced transitions in SCLCP isotropic melts. The purpose of the present paper is to analyze the flow behavior produced above $T_{\mathrm{NI}}$ by a SCLCP whose main-chain conformation is already prolate in the equilibrium nematic phase [7]. The experimental techniques used are nonlinear rheology and flow birefringence. A nonequilibrium phase

\footnotetext{
*Corresponding author.
}

compatible with shear-banding is identified together with the observation of a second nonlinear behavior corresponding to an oscillating regime.

The SCLCP chosen, $\mathrm{PA}_{4}-\mathrm{CN}$, is characterized as a prolate nematic polymer [7]. The monomers have been synthesized at the Laboratoire Léon Brillouin and polymerized by PolymerExpert via controlled radical polymerization. The polymer described here corresponds to a molecular weight of $M_{\mathrm{W}}=85800$ and a polydispersity index of $I=1$. 1 . This molecular weight corresponds to a nonentangled polymer and no rubbery plateau was found in viscoelastic measurements. This $\mathrm{PA}_{4}-\mathrm{CN}$ presents the following succession of mesophases: $\mathrm{Tg}-30{ }^{\circ} \mathrm{C}-\mathrm{N}-116^{\circ} \mathrm{C}-I$ and corresponds to the formula

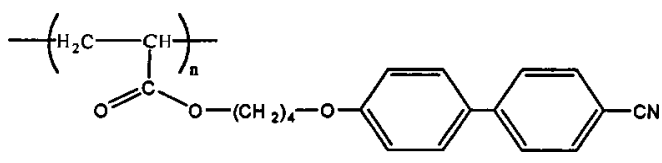

Rheological measurements were performed with a Rheometric Dynamic Analyser (RDA II) using an aluminum cone and plate cell (diameter, $12 \mathrm{~mm}$; angle, $0.17 \mathrm{rad}$ ) and an aluminum plate-plate cell (diameter, $12 \mathrm{~mm}$; gap, $0.5 \mathrm{~mm}$ ) in a controlled shear rate mode. Birefringence measurements were carried out, at various gaps from 60 to $200 \mu \mathrm{m}$, with a custom-improved Linkam setting equipped with a Quartz plate-plate type shear cell.

Figure 1(a) displays the shear stress versus time evolution for different shear rates at $T=122^{\circ} \mathrm{C}\left(\Delta T=T-T_{\mathrm{NI}}=6{ }^{\circ} \mathrm{C}\right.$ above the nematic-isotropic transition). At low shear rates, as long as the flow does not exceed $16 \mathrm{~s}^{-1}$, the stress response is constant; the isotropic melt presents a Newtonian response. For shear rates of 17,18 , and $19 \mathrm{~s}^{-1}$, the stress weakly overshoots at short times and then relaxes (in less than $1000 \mathrm{~s})$ to the same stationary state stress value $\sigma$ $=3900 \mathrm{~Pa}$. This indicates the departure from the Newtonian behavior. The stationary stress versus shear rate curve, measured at the same temperature, is displayed in the inset of Fig. 1(a). The stationary curves are obtained by applying each strain rate for a time $t_{\mathrm{w}}=1000 \mathrm{~s}$, after which the measured stress value is averaged over a time $t_{\mathrm{r}}=100 \mathrm{~s}$. These measurements are independent of the transient ones since the shear rate is incremented without relaxing between two suc- 

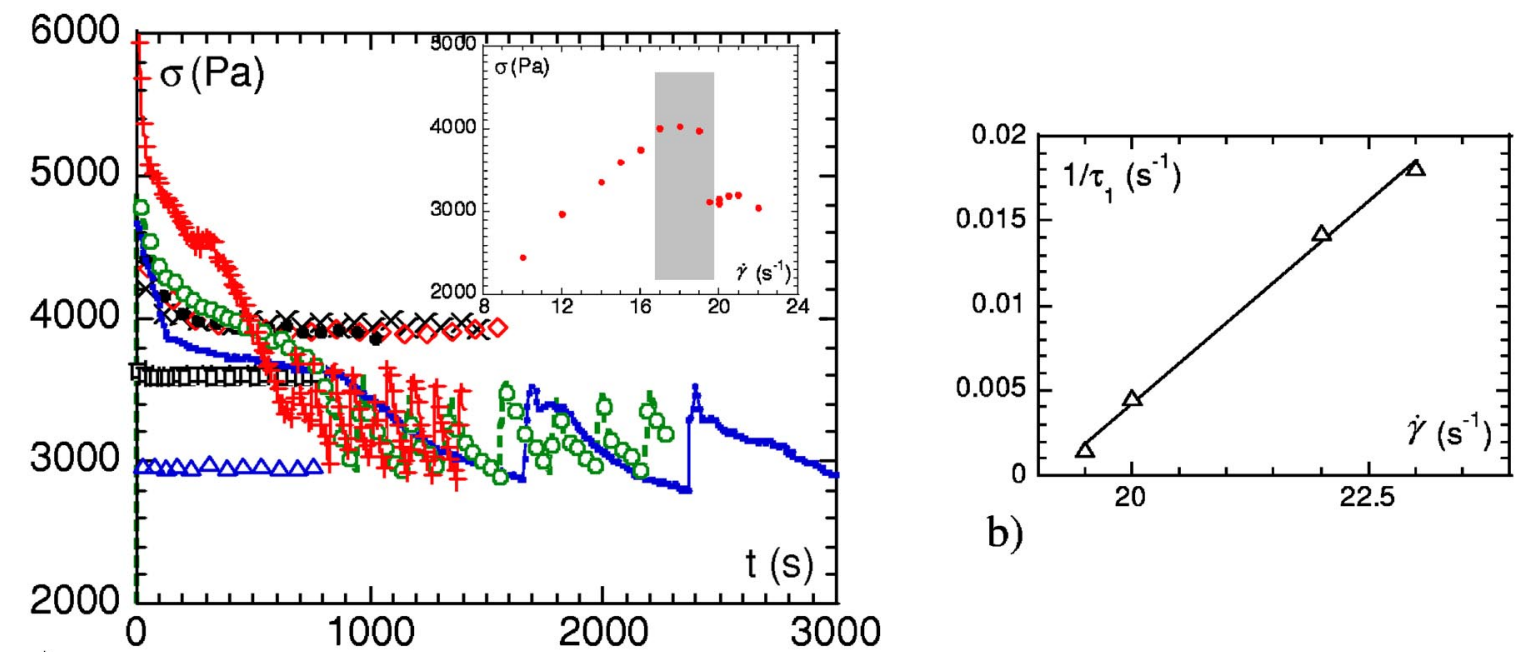

b)

a)
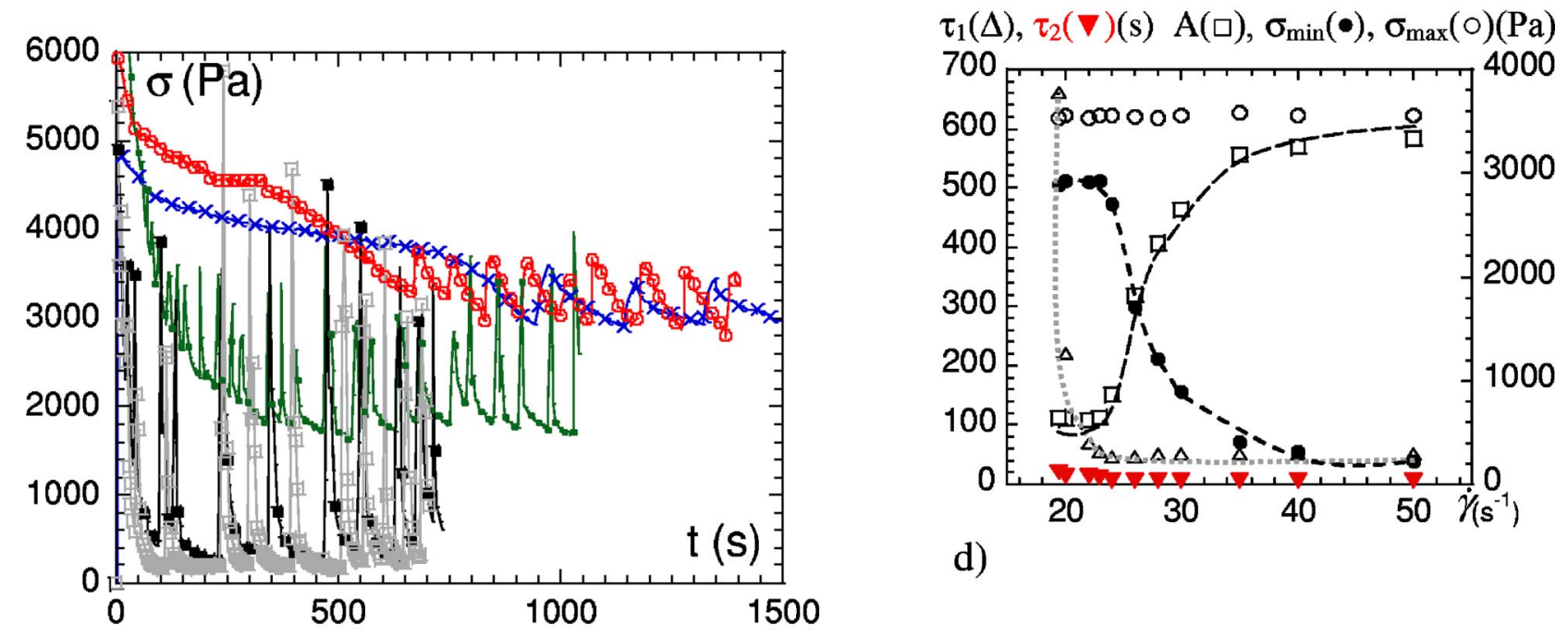

d)

c)

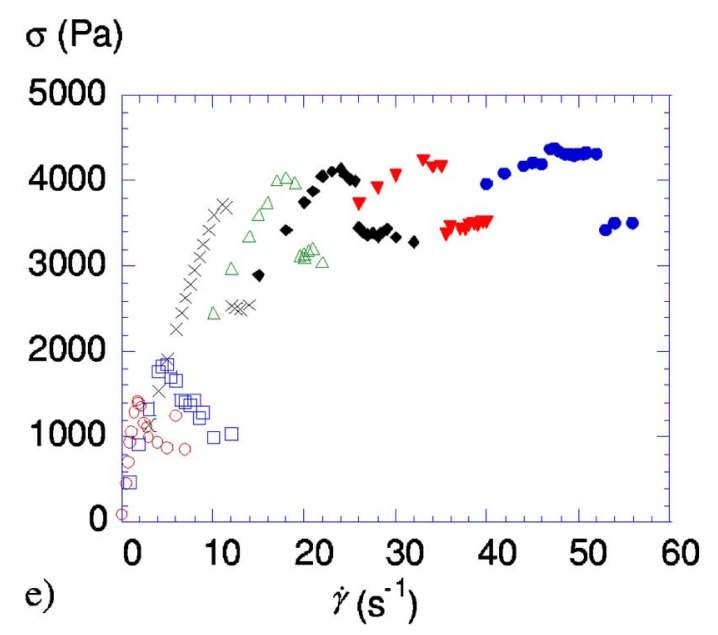

FIG. 1. Nonlinear rheology, in cone-plate geometry, at $T=122{ }^{\circ} \mathrm{C}\left(\Delta T=T-T_{\mathrm{NI}}=6{ }^{\circ} \mathrm{C}\right)$. (a) Shear stress vs time at different shear rates: $\triangle, 12 \mathrm{~s}^{-1} ; \square, 15 \mathrm{~s}^{-1} ; \times, 17 \mathrm{~s}^{-1} ; \diamond, 18 \mathrm{~s}^{-1} ; \mathbf{O}, 19 \mathrm{~s}^{-1} ;-\boldsymbol{-}, 19.5 \mathrm{~s}^{-1} ;--\bigcirc--, 20 \mathrm{~s}^{-1} ;--+-, 22 \mathrm{~s}^{-1}$. The inset shows the stationary shear stress vs shear rate. (b) Dependence of the inverse of the pseudoperiod $\tau_{1}$. (c) Shear stress vs time for $\dot{\gamma}:-X_{-}, 20 \mathrm{~s}^{-1} ;-\mathbf{0}_{-}^{-,} 22 \mathrm{~s}^{-1} ;-, 26 \mathrm{~s}^{-1} ;-\boldsymbol{\square}_{-}$, $40 \mathrm{~s}^{-1} ;-\square-, 50 \mathrm{~s}^{-1}$. (d) Evolution as a function of the applied shear rate of the two characteristic times of the period: $\Delta\left(\tau_{1}\right)$ and $\boldsymbol{\nabla}\left(\tau_{2}\right)$, of the amplitude $\square$ (A) and of the $\bigcirc$ (maximum) and $\bigcirc$ (minimum) stress values of the oscillation. The dotted lines are guides to the eye. (e) Evolution vs shear rate of the stationary shear stress at various temperatures below and above the isotropic-nematic temperature: $\bigcirc$, $\Delta T=-6{ }^{\circ} \mathrm{C} ; \square, \Delta T=-1{ }^{\circ} \mathrm{C} ; \times, \Delta T=+1{ }^{\circ} \mathrm{C} ; \Delta, \Delta T=+6{ }^{\circ} \mathrm{C} ; \diamond, \Delta T=+9{ }^{\circ} \mathrm{C} ; \boldsymbol{\nabla}, \Delta T=+14{ }^{\circ} \mathrm{C} ;$ and $\bullet, \Delta T=+19^{\circ} \mathrm{C}$. 


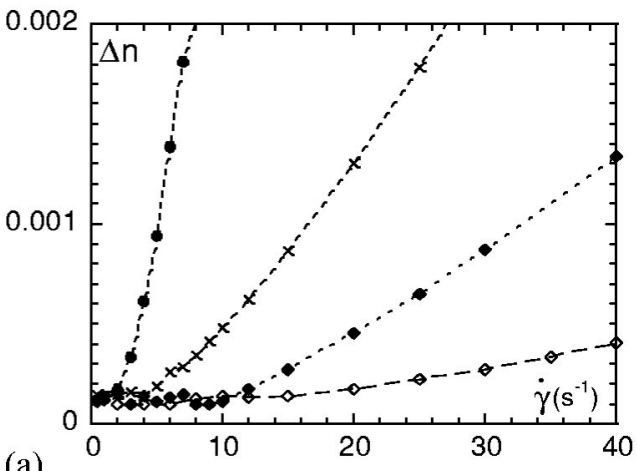

(a)

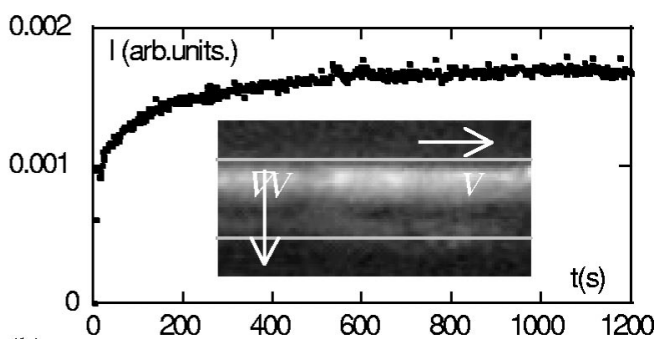

(b)

FIG. 2. Rheomicroscopy measurements: (a) Onset of the nonlinear evolution of the birefringence vs shear rate $(\lambda=470 \mathrm{~nm}$; gap, $100 \mu \mathrm{m})$, measured at different temperatures above the isotropicnematic transition: $\bullet, \Delta T=1{ }^{\circ} \mathrm{C} ; \times, \Delta T=3{ }^{\circ} \mathrm{C} ; \diamond, \Delta T=5{ }^{\circ} \mathrm{C}$; $\diamond, \Delta T=9^{\circ} \mathrm{C}$. (b) Time transient evolution of the transmitted intensity between crossed polarizers at $\dot{\gamma}=30 \mathrm{~s}^{-1}$ and $\Delta T=5^{\circ} \mathrm{C}$. These results are independent of the gap setting from 60 to $200 \mu \mathrm{m}$. The inset illustrates the appearance of a flow birefringence band in the (velocity, gradient of velocity) plane at $\dot{\gamma}=6.5 \mathrm{~s}^{-1}$ and $\Delta T$ $=1{ }^{\circ} \mathrm{C}($ gap, $1 \mathrm{~mm})$.

cessive values. As can be seen [inset of Fig. 1(a)] for $\dot{\gamma}$ $>\dot{\gamma}_{C 1}=17 \mathrm{~s}^{-1}$, the shear stress evolves no longer linearly with the shear rate and a narrow stress plateau is appearing. For the equivalent shear rates, the rheomicroscopy measurements [Fig. 2(a)] show a nonlinear evolution of the flow birefringence. The texture indicates that the induced phase is nematic and of shear aligning type. These results indicate a shear-induced nematic phase transition appearing through a flow birefringence banding process as illustrated in the inset and as already previously observed [3].

At $\dot{\gamma} \geqslant \dot{\gamma}^{*}=19.5 \mathrm{~s}^{-1}$, the shear stress curve presents a new nonlinear behavior: there is at first for a short time, a stress overshoot followed after $1000 \mathrm{~s}$ by the appearance of time-periodic stress variation oscillating around an average stress value of $3200 \mathrm{~Pa}$. The shape of the oscillation is strongly asymmetrical, indicating a two-step process involving two distinct mechanisms: a slow one corresponding to the stress decrease (characterized by the time $\tau_{1}$ ) and a rapid one corresponding to the stress increase $\left(\tau_{2}\right)$.

For applied shear rates between $19.5<\dot{\gamma}<23 \mathrm{~s}^{-1}$, the period of the time-periodic stress oscillations decreases, keeping, however, the same amplitude and defining an apparent second stress plateau as shown in the inset of Fig. 1(a) (the data displayed in the inset at $\dot{\gamma}>\dot{\gamma}^{*}$ correspond to average stress values). The inset of Fig. 1(a) indicates also that this pseudostress plateau is below the stress value at the stress plateau; the oscillating state corresponds thus to a minimized constant stress. Studying the variation of the oscillation period with the imposed shear rate in greater detail, one can find that the rapid time $\tau_{2}$ is invariant and that the inverse of the slow time $\tau_{1}$ follows a linear law with the shear rate as shown in Fig. 1(b). The rapid time $\tau_{2}$ is not illustrated since it remains close to $10 \mathrm{~s}$ whatever the shear rate.

Figure 1(c) presents the evolution of the stress oscillations at $T=122^{\circ} \mathrm{C}$ at higher shear rate. It indicates that a second oscillating regime takes place for shear rates above a new critical shear rate $\dot{\gamma}>\dot{\gamma}^{* *}=23 \mathrm{~s}^{-1}$. This second regime of oscillation is characterized by the invariance of the oscillation period (and so $\tau_{1}$ and $\tau_{2}$ ) and by a decreasing minimum stress value with the applied shear rate keeping however a constant maximum stress value. Figure 1(d) presents the evolution of the different parameters characterizing the oscillations in the two regimes. The crossover between the two regimes is characterized by the beginning of the decrease in the minimum stress value and the conservation of the slow time $\tau_{1}$.

Analogous shear stress behaviors are observed at other temperatures, at least up to $\Delta T=19^{\circ} \mathrm{C}$ above the isotropicnematic transition and also with a plate-plate geometry, displaying first the stress plateau and, at higher shear rates, sustained oscillations. Figure 1(e) illustrates the evolution of the shear stress versus shear rates at various temperatures. One can notice that below the isotropic-nematic transition, shear induced undamped oscillations are also generated but without developing an apparent stress plateau. Finally, no timedependent phenomenon can be noticed by the optical observation of the gap during the experiment.

The stability of the phenomenon with respect to the temperature, to the flow geometry and the long-lived oscillation periods $(>50 \mathrm{~s})$ which are incommensurate with the rotation of the rotor but proportional (for $19.5<\dot{\gamma}<23 \mathrm{~s}^{-1}$ ) to the inverse of a reduced shear rate, point out a mechanism definitively due to genuine physical properties of the material and more particularly related to its ordered state (it does not appear as long as the shear-induced phase is not achieved).

Flow instabilities of different nature may happen when shearing viscoelastic systems [8] or nematics [9]. In the present case, the stress oscillations cannot be assimilated with the establishment of a tumbling regime [10,11], since no damping is observed even after $4000 \mathrm{~s}$ and no time-periodic oscillations are detected in the birefringence experiments [Fig. 2(b)].

Flow-induced oscillating viscosity, in controlled shear stress mode, was reported in the case of lyotropic lamellar phases near a transition from a disordered to an ordered state [10]. These oscillations were related to a hysteresis phenomenon in the stress versus shear rate curve, which is not observed here. Light scattering data indicated that the lyotropic viscosity oscillation corresponds to the time-periodic alternation of two distinct structures. It results in a conservation of amplitude of the viscosity oscillations regardless of the applied stress, the top and the bottom corresponding to the two states. In the SCLCP case, the second state is not developed and there is no alternation of the two phases [12]. Also in the SCLCP case, the absence of flow birefringence oscillations 
[Fig. 2(b)] indicates either that the phenomenon does not appear in the quartz shear cell or that it is below the instrumental resolution. In both cases, it implies that the oscillation does not reflect an intrinsic bulk property (determined by the flow parameters) but a mechanism generated at the boundary and by the anchoring conditions. Now, if the shear stress reaches a value such that the viscous deformation is no longer possible, an interfacial friction state takes place. The energy excess is evacuated in a slip mechanism. The asymmetry of the oscillations evokes typically a stick-slip behavior implying a zone of cohesive rupture $[13,14]$. During the slip process, the measured shear stress is an apparent lowered value in agreement with the observed lower stress value of the pseudoplateau. The mechanism is here complicated by the conjugated properties of both phase and polymer viscoelasticity. In solid-entangled polymer melts or solutions the slip is an expected phenomenon; the conformational entropy of the macromolecule close to the surface is lower than in the bulk [13]. Above a critical shear rate, the chain under tension loses its anchoring contact creating a slip layer on or near the surface of a lower frictional state similar to that of an unentangled polymer fluid [14]. In the stick-slip process, this slip layer relaxes sticking again to the substrate. This stick process together with the stretch phase is at the origin of the shear stress increase in stick-slip phenomena, whereas slipping explains the abrupt stress decrease.

From considerations above and information carried out from our experiments (since during the slip, we do access apparent viscosity and stress values), the following analysis is proposed for the interpretation of the time-dependent oscillation. The abrupt stress increase of the oscillation is identified as the sticking and stretching phase, whereas the slow stress decrease is associated with a progressive disanchoring of the polymer at the interface. The key point in a stick-slip mechanism is that the bulk cohesion forces have to be stronger than the anchoring forces. Indeed, stick-slip is appearing when the flow conditions are no longer fulfilled, i.e., the viscous dissipation is no more enabled and becomes substituted by a friction mechanism. Here, it seems that the nematic-solid interface triggers off the stick-slip mechanism more easily than the isotropic-solid interface one. Indeed, below $T_{\mathrm{NI}}$, the oscillation is appearing immediately above a low critical shear rate, whereas above $T_{\mathrm{NI}}$, the end of the stress plateau should be reached [Fig. 1(e)]. Above $T_{\mathrm{NI}}$, the starting of the oscillating mechanism can be due to an autophobic effect; an anchoring condition change occurs when the nematic phase comes into contact with the solid interface (the nematic phase should therefore fill the entire gap). The stick-slip results also from a cohesive feature, i.e., the nonequilibrium polymer nematic state behaves as a network of elastic links with respect to the interface. The lifetime of the dynamic contact links determines the order of magnitude of the two characteristic times $\tau_{1}$ and $\tau_{2}$ of the oscillation period [15]. These times are long and the stress oscillation amplitude low showing that the anchoring forces are weak; i.e., the melt is not so far away from the static friction state, like a weakly crosslinked network. In the first regime of oscillations defined by constant minimum and maximum stress values, the initial and terminal states are recovered, only the period $\tau_{1}$ changes: this is an elastic regime. The elastic character appears also in the proportionality relationship between the disanchoring time $\left(\tau_{1}\right)$ and the inverse of the reduced shear rate $\left(\dot{\gamma}-\dot{\gamma}^{*}\right)$ [Fig. 1(b)]. It defines a "strain" rate of 210, characterizing a smooth contact breaking [16]. Finally, the independence of the time $\left(\tau_{2}\right)$ with respect to the applied shear rate seems to indicate that the stretch process has a negligible contribution to the stress during the resticking mechanism in agreement with a weak anchoring condition.

The second regime of oscillation, at $\dot{\gamma} \geqslant \dot{\gamma}^{*} \geqslant \dot{\gamma}^{* *}$ $=23 \mathrm{~s}^{-1}$, is interpreted by the superposition of the present stick-slip process, to a continuous modification of the anchoring conditions; the stick-slip mechanism is preserved but a continuous slip appears simultaneously within the sample. This regime is characterized by constant $\tau_{1}$ and $\tau_{2}$ and a decreasing minimum stress value with increasing shear rate. When the applied shear rate is increased, the continuous slip process $\left(\dot{\gamma}_{\text {slip }}\right)$ increases keeping a constant felt shear rate $\dot{\gamma}_{\text {felt }}$ and thus the same "strain" rate; $\dot{\gamma}_{\text {applied }}=\dot{\gamma}_{\text {felt }}$ $+\dot{\gamma}_{\text {slip }}$. In this nonelastic regime, the energy is dissipated via a progressive decrease of the minimum stress value of the oscillation. The ultimate state would imply a total continuous slip.

We have investigated the flow behavior of a SCLCP characterized by a prolate conformation in the equilibrium nematic phase. At $T>T_{\mathrm{NI}}$, the simultaneous appearance, at a first critical shear rate $\dot{\gamma}_{C 1}$, of a flow birefringence and a stress plateau in the shear stress versus shear rate curves, revealed that this polymer presents a nonequilibrium isotropic to shear aligning nematic transition under flow. This result shows that shear-induced $I-N$ transition is a general property of SCLCPs which does not originate from specific main-chain/side chain coupling allowed to undergo oblate to prolate transitions under flow. The study of the transient stress evolution revealed, above a second critical shear rate $\dot{\gamma}^{*}$, a nonlinear behavior characterized by time-periodic flow-induced oscillations. $\mathrm{Bi}$ refringence measurements indicated that bulk or phase mechanisms such as tumbling are excluded revealing the entrance in a nonflow regime (friction) compatible with a stickslip behavior. Two regimes of stress oscillations are identified depending on the applied shear rate. A first regime of elastic response is identified at low shear rate, succeeded by a second regime where a continuous wall slip occurs in addition to the initial stick-slip mechanism. These observations were carried out on a nonentangled SCLCP and in its nonequilibrium nematic state only. It fits the few numerous studies on low viscous materials $[17,18]$ against the consensus that unlike simple liquids, only entangled polymers can and do violate the no-slip condition above a critical stress. The fact that the oscillation takes place once the long range order (nematic phase) is established, indicates that the phase possesses somehow the necessary cohesion to the polymer to allow the slipping. This cohesion condition is also met to explain the surprising stretching ability observed on unentangled nematic SCLCPs [19]. Such features show that shear banding models remain open for discussion. Varying the an- 
choring energy (surface states), the flow conditions, and the characteristics of the material (polymer length in particular) is an essential challenge for understanding of the parameters governing the balance from flow to nonflow conditions in complex fluids.
The authors are very grateful to Hélène Montès for her hospitality and the access to the rheometer at the ESPCI, to J. P. Le Barny and G. Kirsch for advice in chemistry, and to P. Baroni for his assistance during flow birefringence experiments.
[1] J. D. Ferry, Viscoelastic Properties of Polymers (Wiley, New York, 1970).

[2] S. Safinya, E. B. Sirota, and R. J. Plano, Phys. Rev. Lett. 66, 1986 (1991); H. See, M. Doi, and R. Larson, J. Chem. Phys. 92, 792 (1990); P. D. Olmsted and P. Goldbart, Phys. Rev. A 41, 4578 (1990); 46, 4966 (1992).

[3] C. Pujolle-Robic and L. Noirez, Nature (London) 409, 167 (2001); C. Pujolle-Robic, P. D. Olmsted, and L. Noirez, Europhys. Lett. 59, 364 (2002).

[4] V. Schmitt et al., Langmuir 10, 955 (1994); J. F. Berret et al., Europhys. Lett. 25, 521 (1994).

[5] J. F. Berret, G. Porte, and J. P. Decruppe, Phys. Rev. E 55, 1 (1997); E. Cappelaere et al., ibid. 56, 1869 (1997); J. P. Decruppe et al., J. Phys. II 7, 257 (1997); C. Grand, J. Arrault, and M. E. Cates, ibid. 7, 1071 (1997).

[6] D. Collin and P. Martinoty, Physica A 320, 235 (2003).

[7] L. Noirez, P. Keller, and J. P. Cotton, Liq. Cryst. 18, 129 (1995).

[8] R. G. Larson, Rheol. Acta 31, 213 (1992).

[9] P. Oswald and P. Pieranski, Les Cristaux Liquides (Gordon and Breach, New York, 2000); P. Pieranski and E. Guyon, Phys.
Rev. A 9, 404 (1974).

[10] G. Marrucci, Macromolecules 24, 4176 (1991).

[11] I. Quijada-Garrido et al., Macromolecules 33, 3844 (2000).

[12] A. S. Wunenburger et al., Phys. Rev. Lett. 86, 1374 (2001); J. B. Salmon, A. Colin, and D. Roux, Phys. Rev. E 66, 031505 (2002).

[13] S. Asakura and F. Oosawa, J. Chem. Phys. 22, 1255 (1952); E. Dimarzio and F. McCrackin, ibid. 43, 539 (1965); T. T. Dao and L. A. Archer, Langmuir 12, 2616 (2002).

[14] P. G. de Gennes, J. Chem. Phys. 60, 5030 (1974); F. Brochard and P. G. de Gennes, Langmuir 8, 3033 (1992); R. H. Burton, M. J. Folkes, K. A. Narh, and A. Keller, J. Mater. Sci. 18, 315 (1983); V. Mhetar and L. A. Archer, Macromolecules 31, 6639 (1998).

[15] E. Michel, J. Appell, F. Molino, J. Kieffer, and G. Porte, J. Rheol. 45, 1465 (2001).

[16] L. Bocquet and H. J. Jensen, J. Phys. (France) 7, 1603 (1997).

[17] G. Reiter et al., Science 263, 1741 (1994); L. A. Archer et al., Rheol. Acta 36, 579 (1997).

[18] C. Liu and D. J. Pine, Phys. Rev. Lett. 77, 2121 (1996).

[19] V. Fourmeaux-Demange et al., Eur. Phys. J. E 1, 301 (2000). 